\title{
On Weakly Associative Lattices and Near Lattices
}

\author{
Damian Sawicki \\ Institute of Informatics \\ University of Białystok \\ Poland
}

\author{
Adam Grabowski \\ Institute of Informatics \\ University of Białystok \\ Poland
}

\begin{abstract}
Summary. The main aim of this article is to introduce formally two generalizations of lattices, namely weakly associative lattices and near lattices, which can be obtained from the former by certain weakening of the usual well-known axioms. We show selected propositions devoted to weakly associative lattices and near lattices from Chapter 6 of [15, dealing also with alternative versions of classical axiomatizations. Some of the results were proven in the Mizar [1, 2] system with the help of Prover9 [14] proof assistant.
\end{abstract}

MSC: 68V20 06B05 06B75

Keywords: weakly associative lattice; near lattice

MML identifier: LATWAL_1, version: 8.1.11 5.66.1402

\section{INTRODUCTION}

Lattice theory is widely represented in the Mizar Mathematical Library, with Żukowski's first article [18, following Birkhoff [3] and Grätzer [11, [12]. In parallel, the theory of partially ordered sets was developed [4] treated generally as relational structures, even if informally the notions are quite similar [9], [7]. The review of the mechanization of lattice theory in Mizar, with the example of the solution of the Robbins problem, is contained in [6].

Our work can be seen as a step towards a Mizar support for [15] or [16], where original proof objects by OTTER/Prover9 were used. Some preliminary works in this direction were already done in [8] by present authors. We use the interface ott2miz [17] which allows for the automated translation of proofs; 
these automatically generated proofs are usually quite lengthy, even after native enhancements done by internal Mizar software for library revisions.

Weakly associative lattices were studied in [5]. In the present development, we deal with the parts of Chap. 6 "Lattice-like algebras" of [15], pp. 111-135, devoted to this class of lattices. In this sense, we continue the work started by Kulesza and Grabowski in [13, devoted to the formalization of quasi-lattices.

The class of weakly associative lattices (or WA-lattices, WAL) can be characterized from the standard set of axioms for lattices (with idempotence for the join and meet operations included), where the ordinary associative laws are replaced by the so-called part-preservation laws. The characteristic axiom is however W3 (or, dual W3' - compare Def. 1 and Def. 2). Section 2 contains also equivalent formulation of these axioms, using ordering relation on lattices. The earlier seems to be a bit more feasible taking into account the role of equality in the Mizar system [10] and the design of Prover9.

In Section 3 we show how described binary lattice operations can be associated with the corresponding ordering relation. Obviously, the associativity can only be shown under some conditions for elements (see theorems (15) and (16)). If we assume distributivity, the relation is transitive, as in usual lattices. Section 4 contains the proof that adding the distributivity condition to the set of usual WAL axioms, the associativity can be proven.

Then we deal with another generalization of lattices, i.e. near lattices (absorption law is weakened). Def. 6 and Def. 7 provide standard examples of these structures which are not necessarily lattices (see Def. 10 for the definition of the structure). The lattice operations are given by

\begin{tabular}{|c|c|c|c|c|c|c|}
\hline Ј & 0 & 1 & 2 & $\sqcap$ & 0 & 1 \\
\hline 0 & 0 & 1 & 0 & 0 & 0 & 0 \\
\hline 1 & 1 & 1 & 2 & 1 & 0 & 1 \\
\hline 2 & 0 & 2 & 2 & 2 & 2 & 1 \\
\hline
\end{tabular}

Associativity laws do not hold here, so this is not a lattice.

The rest of the article is devoted to alternative axiomatizations of WAL. WAL-3 - equivalent set of axioms describing WAL is expressed in the form of five separate attributes to make Mizar registrations mechanism working (see Def. 11-Def. 15). It is shown that these adjectives imply the standard attributes for lattices.

In Section 8 WAL-4 is defined (the short sigle axiom system for WAL). We conclude with the proof if WAL-4 holds, then lattice operations are idempotent.

Some of the proofs were produced by means of Prover9, so they are definitely lengthy. The enhancement of the lemmas, including their shortening, reorganization and clustering, can be interesting and useful future work. 


\section{Preliminaries}

From now on $L$ denotes a non empty lattice structure and $v_{100}, v_{102}, v_{2}, v_{1}$, $v_{0}, v_{3}, v_{101}$ denote elements of $L$.

Let us consider $v_{0}, v_{1}$, and $v_{2}$. Now we state the propositions:

(1) Suppose for every $v_{0}, v_{0} \sqcap v_{0}=v_{0}$ and for every $v_{1}$ and $v_{0}, v_{0} \sqcap v_{1}=v_{1} \sqcap v_{0}$ and for every $v_{0}, v_{0} \sqcup v_{0}=v_{0}$ and for every $v_{1}$ and $v_{0}, v_{0} \sqcup v_{1}=v_{1} \sqcup v_{0}$ and for every $v_{2}, v_{1}$, and $v_{0},\left(\left(v_{0} \sqcup v_{1}\right) \sqcap\left(v_{2} \sqcup v_{1}\right)\right) \sqcap v_{1}=v_{1}$ and for every $v_{2}, v_{1}$, and $v_{0},\left(\left(v_{0} \sqcap v_{1}\right) \sqcup\left(v_{2} \sqcap v_{1}\right)\right) \sqcup v_{1}=v_{1}$ and for every $v_{1}, v_{2}$, and $v_{0}, v_{0} \sqcap\left(v_{1} \sqcup\left(v_{0} \sqcup v_{2}\right)\right)=v_{0}$. Then $\left(v_{0} \sqcap v_{1}\right) \sqcap v_{2}=v_{0} \sqcap\left(v_{1} \sqcap v_{2}\right)$.

(2) Suppose for every $v_{0}, v_{0} \sqcap v_{0}=v_{0}$ and for every $v_{1}$ and $v_{0}, v_{0} \sqcap v_{1}=v_{1} \sqcap v_{0}$ and for every $v_{0}, v_{0} \sqcup v_{0}=v_{0}$ and for every $v_{1}$ and $v_{0}, v_{0} \sqcup v_{1}=v_{1} \sqcup v_{0}$ and for every $v_{2}, v_{1}$, and $v_{0},\left(\left(v_{0} \sqcup v_{1}\right) \sqcap\left(v_{2} \sqcup v_{1}\right)\right) \sqcap v_{1}=v_{1}$ and for every $v_{2}, v_{1}$, and $v_{0},\left(\left(v_{0} \sqcap v_{1}\right) \sqcup\left(v_{2} \sqcap v_{1}\right)\right) \sqcup v_{1}=v_{1}$ and for every $v_{1}, v_{2}$, and $v_{0}, v_{0} \sqcap\left(v_{1} \sqcup\left(v_{0} \sqcup v_{2}\right)\right)=v_{0}$. Then $\left(v_{0} \sqcup v_{1}\right) \sqcup v_{2}=v_{0} \sqcup\left(v_{1} \sqcup v_{2}\right)$.

Let us consider $v_{1}$ and $v_{2}$. Now we state the propositions:

(3) Suppose for every $v_{0}, v_{0} \sqcup v_{0}=v_{0}$ and for every $v_{1}, v_{2}$, and $v_{0}, v_{0} \sqcap\left(v_{1} \sqcup\right.$ $\left.\left(v_{0} \sqcup v_{2}\right)\right)=v_{0}$. Then $v_{1} \sqcap\left(v_{1} \sqcup v_{2}\right)=v_{1}$.

(4) Suppose for every $v_{1}$ and $v_{0}, v_{0} \sqcap v_{1}=v_{1} \sqcap v_{0}$ and for every $v_{0}, v_{0} \sqcup v_{0}=v_{0}$ and for every $v_{1}$ and $v_{0}, v_{0} \sqcup v_{1}=v_{1} \sqcup v_{0}$ and for every $v_{2}, v_{1}$, and $v_{0}$, $\left(\left(v_{0} \sqcap v_{1}\right) \sqcup\left(v_{2} \sqcap v_{1}\right)\right) \sqcup v_{1}=v_{1}$. Then $v_{1} \sqcup\left(v_{1} \sqcap v_{2}\right)=v_{1}$.

\section{Definition of Attributes}

Let $L$ be a non empty lattice structure. We say that $L$ is satisfying W3 if and only if

(Def. 1) for every elements $v_{2}, v_{1}, v_{0}$ of $L,\left(\left(v_{0} \sqcup v_{1}\right) \sqcap\left(v_{2} \sqcup v_{1}\right)\right) \sqcap v_{1}=v_{1}$.

We say that $L$ is satisfying W3' if and only if

(Def. 2) for every elements $v_{2}, v_{1}, v_{0}$ of $L,\left(\left(v_{0} \sqcap v_{1}\right) \sqcup\left(v_{2} \sqcap v_{1}\right)\right) \sqcup v_{1}=v_{1}$.

Let $L$ be a meet-absorbing, join-absorbing, meet-commutative, non empty lattice structure. Let us note that $L$ is satisfying W3 if and only if the condition (Def. 3) is satisfied.

(Def. 3) for every elements $v_{2}, v_{1}, v_{0}$ of $L, v_{1} \sqsubseteq\left(v_{0} \sqcup v_{1}\right) \sqcap\left(v_{2} \sqcup v_{1}\right)$.

Let us consider $L$. Observe that $L$ is satisfying W3' if and only if the condition (Def. 4) is satisfied.

(Def. 4) for every $v_{2}, v_{1}$, and $v_{0},\left(v_{0} \sqcap v_{1}\right) \sqcup\left(v_{2} \sqcap v_{1}\right) \sqsubseteq v_{1}$. 
Let us note that every non empty lattice structure which is meet-commutative, join-idempotent, join-commutative, and satisfying W3' is also quasi-meet-absorbing and every non empty lattice structure which is meet-commutative, meetidempotent, join-commutative, and satisfying W3 is also join-absorbing and every non empty lattice structure which is trivial is also satisfying W3' and there exists a non empty lattice structure which is satisfying W3, satisfying W3', join-idempotent, meet-idempotent, join-commutative, and meet-commutative.

A weakly associative lattice is a join-idempotent, meet-idempotent, joincommutative, meet-commutative, satisfying W3, satisfying W3', non empty lattice structure.

A WA-lattice is a weakly associative lattice. Note that every join-associative, meet-absorbing lattice is satisfying W3'.

Let $L$ be a non empty lattice structure. We say that $L$ is satisfying WA if and only if

(Def. 5) for every elements $x, y, z$ of $L, x \sqcap(y \sqcup(x \sqcup z))=x$.

\section{On the Ordering Relation Generated by Weakly Associated LATTICES}

Let us note that every non empty lattice structure which is quasi-meetabsorbing, meet-commutative, and join-commutative is also meet-absorbing and every WA-lattice is meet-absorbing.

From now on $L$ denotes a WA-lattice and $x, y, z, u$ denote elements of $L$.

Now we state the propositions:

(5) $x \sqcup y=y$ if and only if $x \sqsubseteq y$.

(6) $x \sqcap y=x$ if and only if $x \sqsubseteq y$.

(7) $x \sqsubseteq x$.

(8) If $x \sqsubseteq y$ and $y \sqsubseteq x$, then $x=y$.

(9) $x \sqsubseteq x \sqcup y$.

(10) $x \sqcap y \sqsubseteq x$.

(11) If $x \sqsubseteq z$ and $y \sqsubseteq z$, then $x \sqcup y \sqsubseteq z$.

(12) There exists $z$ such that

(i) $x \sqsubseteq z$, and

(ii) $y \sqsubseteq z$, and

(iii) for every $u$ such that $x \sqsubseteq u$ and $y \sqsubseteq u$ holds $z \sqsubseteq u$.

The theorem is a consequence of (11) and (9).

(13) If $z \sqsubseteq x$ and $z \sqsubseteq y$, then $z \sqsubseteq x \sqcap y$. 
(14) There exists $z$ such that

(i) $z \sqsubseteq x$, and

(ii) $z \sqsubseteq y$, and

(iii) for every $u$ such that $u \sqsubseteq x$ and $u \sqsubseteq y$ holds $u \sqsubseteq z$.

The theorem is a consequence of (13) and (10).

(15) If $x \sqsubseteq z$ and $y \sqsubseteq z$, then $(x \sqcup y) \sqcup z=x \sqcup(y \sqcup z)$.

(16) If $z \sqsubseteq x$ and $z \sqsubseteq y$, then $(x \sqcap y) \sqcap z=x \sqcap(y \sqcap z)$.

(17) If $L$ is distributive and $x \sqsubseteq y \sqsubseteq z$, then $x \sqsubseteq z$.

\section{Distributivity Implies Associativity}

From now on $L$ denotes a non empty lattice structure and $v_{0}, v_{1}, v_{2}$ denote elements of $L$.

Now we state the proposition:

(18) Suppose for every $v_{0}, v_{0} \sqcap v_{0}=v_{0}$ and for every $v_{1}$ and $v_{0}, v_{0} \sqcap v_{1}=v_{1} \sqcap v_{0}$ and for every $v_{0}, v_{0} \sqcup v_{0}=v_{0}$ and for every $v_{1}$ and $v_{0}, v_{0} \sqcup v_{1}=v_{1} \sqcup v_{0}$ and for every $v_{2}, v_{1}$, and $v_{0},\left(\left(v_{0} \sqcup v_{1}\right) \sqcap\left(v_{2} \sqcup v_{1}\right)\right) \sqcap v_{1}=v_{1}$ and for every $v_{2}, v_{1}$, and $v_{0},\left(\left(v_{0} \sqcap v_{1}\right) \sqcup\left(v_{2} \sqcap v_{1}\right)\right) \sqcup v_{1}=v_{1}$ and for every $v_{1}$ and $v_{0}, v_{0} \sqcap\left(v_{0} \sqcup v_{1}\right)=v_{0}$ and for every $v_{0}, v_{2}$, and $v_{1}, v_{0} \sqcup\left(v_{1} \sqcap v_{2}\right)=$ $\left(v_{0} \sqcup v_{1}\right) \sqcap\left(v_{0} \sqcup v_{2}\right) .\left(v_{0} \sqcup v_{1}\right) \sqcup v_{2}=v_{0} \sqcup\left(v_{1} \sqcup v_{2}\right)$.

Observe that every WA-lattice which is distributive' is also join-associative.

\section{NeAR Lattices}

Let $x, y$ be elements of $\{0,1,2\}$. The functors: $x \sqcap_{\mathrm{N} L} y$ and $x \sqcup_{\mathrm{N} L} y$ yielding elements of $\{0,1,2\}$ are defined by terms

(Def. 6) $\begin{cases}2, & \text { if } x=0 \text { and } y=2 \text { or } x=2 \text { and } y=0, \\ \min (x, y), & \text { otherwise, }\end{cases}$

(Def. 7) $\begin{cases}0, & \text { if } x=0 \text { and } y=2 \text { or } x=2 \text { and } y=0, \\ \max (x, y), & \text { otherwise, }\end{cases}$

respectively. The functors: $\sqcup_{\mathrm{N} L}$ and $\sqcap_{\mathrm{N} L}$ yielding binary operations on $\{0,1,2\}$ are defined by conditions

(Def. 8) for every elements $x, y$ of $\{0,1,2\}, \sqcup_{\mathrm{N} L}(x, y)=x \sqcup_{\mathrm{N} L} y$,

(Def. 9) for every elements $x, y$ of $\{0,1,2\}, \sqcap_{\mathrm{N} L}(x, y)=x \sqcap_{\mathrm{N} L} y$, respectively. 


\section{Examples of Near Lattices}

The functor ExNearLattice yielding a non empty lattice structure is defined by the term

(Def. 10) $\left\langle\{0,1,2\}, \sqcup_{\mathrm{N} L}, \sqcap_{\mathrm{N} L}\right\rangle$.

One can check that ExNearLattice is non join-associative and non meetassociative and every non empty lattice structure which is trivial is also meetidempotent, join-commutative, quasi-meet-absorbing, and join-absorbing.

A near lattice is a join-idempotent, meet-idempotent, join-commutative, meet-commutative, quasi-meet-absorbing, join-absorbing, non empty lattice structure.

One can check that ExNearLattice is join-commutative, meet-commutative, join-idempotent, meet-idempotent, join-absorbing, and meet-absorbing and every join-commutative, meet-commutative, non empty lattice structure which is meet-absorbing is also quasi-meet-absorbing and every join-commutative, meetcommutative, non empty lattice structure which is quasi-meet-absorbing is also meet-absorbing.

Now we state the proposition:

(19) (i) ExNearLattice is a near lattice, and

(ii) ExNearLattice is not a lattice.

\section{Alternative Axioms for WAL}

From now on $L$ denotes a non empty lattice structure and $v_{101}, v_{100}, v_{2}, v_{1}$, $v_{0}, v_{102}, v_{103}, v_{3}$ denote elements of $L$.

Now we state the proposition:

(20) Suppose for every $v_{1}$ and $v_{0},\left(v_{0} \sqcap v_{1}\right) \sqcup\left(v_{0} \sqcap\left(v_{0} \sqcup v_{1}\right)\right)=v_{0}$ and for every $v_{0}$ and $v_{1},\left(v_{0} \sqcap v_{0}\right) \sqcup\left(v_{1} \sqcap\left(v_{0} \sqcup v_{0}\right)\right)=v_{0}$ and for every $v_{1}$ and $v_{0}$, $\left(v_{0} \sqcap v_{1}\right) \sqcup\left(v_{1} \sqcap\left(v_{0} \sqcup v_{1}\right)\right)=v_{1}$ and for every $v_{2}, v_{1}$, and $v_{0},\left(\left(v_{0} \sqcup v_{1}\right) \sqcap\left(v_{2} \sqcup\right.\right.$ $\left.\left.v_{0}\right)\right) \sqcap v_{0}=v_{0}$ and for every $v_{2}, v_{1}$, and $v_{0},\left(\left(v_{0} \sqcap v_{1}\right) \sqcup\left(v_{2} \sqcap v_{0}\right)\right) \sqcup v_{0}=v_{0}$. $v_{0} \sqcup v_{0}=v_{0}$.

Let us consider $v_{0}$ and $v_{1}$. Now we state the propositions:

(21) Suppose for every $v_{1}$ and $v_{0},\left(v_{0} \sqcap v_{1}\right) \sqcup\left(v_{0} \sqcap\left(v_{0} \sqcup v_{1}\right)\right)=v_{0}$ and for every $v_{0}$ and $v_{1},\left(v_{0} \sqcap v_{0}\right) \sqcup\left(v_{1} \sqcap\left(v_{0} \sqcup v_{0}\right)\right)=v_{0}$ and for every $v_{1}$ and $v_{0}$, $\left(v_{0} \sqcap v_{1}\right) \sqcup\left(v_{1} \sqcap\left(v_{0} \sqcup v_{1}\right)\right)=v_{1}$ and for every $v_{2}, v_{1}$, and $v_{0},\left(\left(v_{0} \sqcup v_{1}\right) \sqcap\left(v_{2} \sqcup\right.\right.$ $\left.\left.v_{0}\right)\right) \sqcap v_{0}=v_{0}$ and for every $v_{2}, v_{1}$, and $v_{0},\left(\left(v_{0} \sqcap v_{1}\right) \sqcup\left(v_{2} \sqcap v_{0}\right)\right) \sqcup v_{0}=v_{0}$. Then $v_{0} \sqcap v_{1}=v_{1} \sqcap v_{0}$. The theorem is a consequence of (24). 
(22) Suppose for every $v_{1}$ and $v_{0},\left(v_{0} \sqcap v_{1}\right) \sqcup\left(v_{0} \sqcap\left(v_{0} \sqcup v_{1}\right)\right)=v_{0}$ and for every $v_{0}$ and $v_{1},\left(v_{0} \sqcap v_{0}\right) \sqcup\left(v_{1} \sqcap\left(v_{0} \sqcup v_{0}\right)\right)=v_{0}$ and for every $v_{1}$ and $v_{0}$, $\left(v_{0} \sqcap v_{1}\right) \sqcup\left(v_{1} \sqcap\left(v_{0} \sqcup v_{1}\right)\right)=v_{1}$ and for every $v_{2}, v_{1}$, and $v_{0},\left(\left(v_{0} \sqcup v_{1}\right) \sqcap\left(v_{2} \sqcup\right.\right.$ $\left.\left.v_{0}\right)\right) \sqcap v_{0}=v_{0}$ and for every $v_{2}, v_{1}$, and $v_{0},\left(\left(v_{0} \sqcap v_{1}\right) \sqcup\left(v_{2} \sqcap v_{0}\right)\right) \sqcup v_{0}=v_{0}$. Then $v_{0} \sqcup v_{1}=v_{1} \sqcup v_{0}$. The theorem is a consequence of (24) and (21).

Let $L$ be a non empty lattice structure. We say that $L$ is satisfying WAL- $3_{1}$ if and only if

(Def. 11) for every elements $v_{1}, v_{0}$ of $L,\left(v_{0} \sqcap v_{1}\right) \sqcup\left(v_{0} \sqcap\left(v_{0} \sqcup v_{1}\right)\right)=v_{0}$.

We say that $L$ is satisfying WAL- $3_{2}$ if and only if

(Def. 12) for every elements $v_{0}, v_{1}$ of $L,\left(v_{0} \sqcap v_{0}\right) \sqcup\left(v_{1} \sqcap\left(v_{0} \sqcup v_{0}\right)\right)=v_{0}$.

We say that $L$ is satisfying WAL- $3_{3}$ if and only if

(Def. 13) for every elements $v_{1}, v_{0}$ of $L,\left(v_{0} \sqcap v_{1}\right) \sqcup\left(v_{1} \sqcap\left(v_{0} \sqcup v_{1}\right)\right)=v_{1}$.

We say that $L$ is satisfying WAL- $3_{4}$ if and only if

(Def. 14) for every elements $v_{2}, v_{1}, v_{0}$ of $L,\left(\left(v_{0} \sqcup v_{1}\right) \sqcap\left(v_{2} \sqcup v_{0}\right)\right) \sqcap v_{0}=v_{0}$.

We say that $L$ is satisfying WAL- $3_{5}$ if and only if

(Def. 15) for every elements $v_{2}, v_{1}, v_{0}$ of $L,\left(\left(v_{0} \sqcap v_{1}\right) \sqcup\left(v_{2} \sqcap v_{0}\right)\right) \sqcup v_{0}=v_{0}$.

Let us note that every non empty lattice structure which is trivial is also satisfying WAL- $3_{1}$, satisfying WAL- $3_{2}$, satisfying WAL- $3_{3}$, satisfying WAL- $3_{4}$, and satisfying WAL- $3_{5}$ and every non empty lattice structure which is satisfying WAL- $3_{1}$, satisfying WAL- $3_{2}$, satisfying WAL- $3_{3}$, satisfying WAL- $3_{4}$, and satisfying WAL- $3_{5}$ is also join-idempotent, meet-idempotent, join-commutative, and meet-commutative.

\section{Short Single Axiom for WAL}

Let $L$ be a non empty lattice structure. We say that $L$ is satisfying WAL-4 if and only if

(Def. 16) for every elements $v_{2}, v_{0}, v_{5}, v_{4}, v_{3}, v_{1}$ of $L,\left(\left(\left(v_{0} \sqcap v_{1}\right) \sqcup\left(v_{1} \sqcap\left(v_{0} \sqcup v_{1}\right)\right)\right) \sqcap\right.$ $\left.v_{2}\right) \sqcup\left(\left(\left(v_{0} \sqcap\left(\left(\left(v_{1} \sqcap v_{3}\right) \sqcup\left(v_{4} \sqcap v_{1}\right)\right) \sqcup v_{1}\right)\right) \sqcup\left(\left(\left(v_{1} \sqcap\left(\left(\left(v_{1} \sqcup v_{3}\right) \sqcap\left(v_{4} \sqcup v_{1}\right)\right) \sqcap\right.\right.\right.\right.\right.\right.$ $\left.\left.\left.v_{1}\right)\right) \sqcup\left(v_{5} \sqcap\left(v_{1} \sqcup\left(\left(\left(v_{1} \sqcup v_{3}\right) \sqcap\left(v_{4} \sqcup v_{1}\right)\right) \sqcap v_{1}\right)\right)\right)\right) \sqcap\left(v_{0} \sqcup\left(\left(\left(v_{1} \sqcap v_{3}\right) \sqcup\left(v_{4} \sqcap\right.\right.\right.\right.$ $\left.\left.\left.\left.\left.\left.v_{1}\right)\right) \sqcup v_{1}\right)\right)\right) \sqcap\left(\left(\left(v_{0} \sqcap v_{1}\right) \sqcup\left(v_{1} \sqcap\left(v_{0} \sqcup v_{1}\right)\right)\right) \sqcup v_{2}\right)\right)=v_{1}$.

From now on $L$ denotes a non empty lattice structure and $v_{108}, v_{107}, v_{106}$, $v_{101}, v_{10}, v_{9}, v_{8}, v_{7}, v_{6}, v_{105}, v_{102}, v_{100}, v_{104}, v_{103}, v_{5}, v_{4}, v_{3}, v_{2}, v_{1}, v_{0}$ denote elements of $L$.

Let us consider $v_{0}$. Now we state the propositions:

(23) Suppose for every $v_{2}, v_{0}, v_{5}, v_{4}, v_{3}$, and $v_{1},\left(\left(\left(v_{0} \sqcap v_{1}\right) \sqcup\left(v_{1} \sqcap\left(v_{0} \sqcup v_{1}\right)\right)\right) \sqcap\right.$ $\left.v_{2}\right) \sqcup\left(\left(\left(v_{0} \sqcap\left(\left(\left(v_{1} \sqcap v_{3}\right) \sqcup\left(v_{4} \sqcap v_{1}\right)\right) \sqcup v_{1}\right)\right) \sqcup\left(\left(\left(v_{1} \sqcap\left(\left(\left(v_{1} \sqcup v_{3}\right) \sqcap\left(v_{4} \sqcup v_{1}\right)\right) \sqcap\right.\right.\right.\right.\right.\right.$ 


$$
\begin{aligned}
& \left.\left.\left.v_{1}\right)\right) \sqcup\left(v_{5} \sqcap\left(v_{1} \sqcup\left(\left(\left(v_{1} \sqcup v_{3}\right) \sqcap\left(v_{4} \sqcup v_{1}\right)\right) \sqcap v_{1}\right)\right)\right)\right) \sqcap\left(v _ { 0 } \sqcup \left(\left(( v _ { 1 } \sqcap v _ { 3 } ) \sqcup \left(v_{4} \sqcap\right.\right.\right.\right. \\
& \left.\left.\left.\left.\left.\left.v_{1}\right)\right) \sqcup v_{1}\right)\right)\right) \sqcap\left(\left(\left(v_{0} \sqcap v_{1}\right) \sqcup\left(v_{1} \sqcap\left(v_{0} \sqcup v_{1}\right)\right)\right) \sqcup v_{2}\right)\right)=v_{1} . \text { Then } v_{0} \sqcap v_{0}=v_{0} .
\end{aligned}
$$

(24) Suppose for every $v_{2}, v_{0}, v_{5}, v_{4}, v_{3}$, and $v_{1},\left(\left(\left(v_{0} \sqcap v_{1}\right) \sqcup\left(v_{1} \sqcap\left(v_{0} \sqcup v_{1}\right)\right)\right) \sqcap\right.$ $\left.v_{2}\right) \sqcup\left(\left(\left(v_{0} \sqcap\left(\left(\left(v_{1} \sqcap v_{3}\right) \sqcup\left(v_{4} \sqcap v_{1}\right)\right) \sqcup v_{1}\right)\right) \sqcup\left(\left(\left(v_{1} \sqcap\left(\left(\left(v_{1} \sqcup v_{3}\right) \sqcap\left(v_{4} \sqcup v_{1}\right)\right) \sqcap\right.\right.\right.\right.\right.\right.$ $\left.\left.\left.v_{1}\right)\right) \sqcup\left(v_{5} \sqcap\left(v_{1} \sqcup\left(\left(\left(v_{1} \sqcup v_{3}\right) \sqcap\left(v_{4} \sqcup v_{1}\right)\right) \sqcap v_{1}\right)\right)\right)\right) \sqcap\left(v_{0} \sqcup\left(\left(\left(v_{1} \sqcap v_{3}\right) \sqcup\left(v_{4} \sqcap\right.\right.\right.\right.$ $\left.\left.\left.\left.\left.\left.v_{1}\right)\right) \sqcup v_{1}\right)\right)\right) \sqcap\left(\left(\left(v_{0} \sqcap v_{1}\right) \sqcup\left(v_{1} \sqcap\left(v_{0} \sqcup v_{1}\right)\right)\right) \sqcup v_{2}\right)\right)=v_{1}$. Then $v_{0} \sqcup v_{0}=v_{0}$. The theorem is a consequence of $(23)$.

One can check that every non empty lattice structure which is trivial is also satisfying WAL-4 and every non empty lattice structure which is satisfying WAL-4 is also join-idempotent and meet-idempotent.

\section{REFERENCES}

[1] Grzegorz Bancerek, Czesław Byliński, Adam Grabowski, Artur Korniłowicz, Roman Matuszewski, Adam Naumowicz, Karol Pąk, and Josef Urban. Mizar: State-of-the-art and beyond. In Manfred Kerber, Jacques Carette, Cezary Kaliszyk, Florian Rabe, and Volker Sorge, editors, Intelligent Computer Mathematics, volume 9150 of Lecture Notes in Computer Science, pages 261-279. Springer International Publishing, 2015. ISBN 978-3319-20614-1. doi 10.1007/978-3-319-20615-8_17.

[2] Grzegorz Bancerek, Czesław Byliński, Adam Grabowski, Artur Korniłowicz, Roman Matuszewski, Adam Naumowicz, and Karol Pąk. The role of the Mizar Mathematical Library for interactive proof development in Mizar. Journal of Automated Reasoning, 61(1):9-32, 2018. do1:10.1007/s10817-017-9440-6

[3] Garrett Birkhoff. Lattice Theory. Providence, Rhode Island, New York, 1967.

[4] B.A. Davey and H.A. Priestley. Introduction to Lattices and Order. Cambridge University Press, 2002.

[5] Ervin Fried and George Grätzer. Some examples of weakly associative lattices. Colloquium Mathematicum, 27:215-221, 1973. doi $10.4064 / \mathrm{cm}-27-2-215-221$

[6] Adam Grabowski. Mechanizing complemented lattices within Mizar system. Journal of Automated Reasoning, 55:211-221, 2015. doi:10.1007/s10817-015-9333-5.

[7] Adam Grabowski and Markus Moschner. Managing heterogeneous theories within a mathematical knowledge repository. In Andrea Asperti, Grzegorz Bancerek, and Andrzej Trybulec, editors, Mathematical Knowledge Management Proceedings, volume 3119 of Lecture Notes in Computer Science, pages 116-129. Springer, 2004. doi:10.1007/978-3540-27818-4_9. 3rd International Conference on Mathematical Knowledge Management, Bialowieza, Poland, Sep. 19-21, 2004.

[8] Adam Grabowski and Damian Sawicki. On two alternative axiomatizations of lattices by McKenzie and Sholander. Formalized Mathematics, 26(2):193-198, 2018. doi:10.2478/forma-2018-0017.

[9] Adam Grabowski and Christoph Schwarzweller. Translating mathematical vernacular into knowledge repositories. In Michael Kohlhase, editor, Mathematical Knowledge Management, volume 3863 of Lecture Notes in Computer Science, pages 49-64. Springer, 2006. doi:https://doi.org/10.1007/11618027_4 4th International Conference on Mathematical Knowledge Management, Bremen, Germany, MKM 2005, July 15-17, 2005, Revised Selected Papers.

[10] Adam Grabowski, Artur Korniłowicz, and Christoph Schwarzweller. Equality in computer proof-assistants. In Ganzha, Maria and Maciaszek, Leszek and Paprzycki, Marcin, editor, Proceedings of the 2015 Federated Conference on Computer Science and Information Systems, volume 5 of ACSIS-Annals of Computer Science and Information Systems, pages 45-54. IEEE, 2015. doi 10.15439/2015F229.

[11] George Grätzer. General Lattice Theory. Academic Press, New York, 1978.

[12] George Grätzer. Lattice Theory: Foundation. Birkhäuser, 2011. 
[13] Dominik Kulesza and Adam Grabowski. Formalization of quasilattices. Formalized Mathematics, 28(2):217-225, 2020. doi 10.2478/forma-2020-0019.

[14] William McCune. Prover9 and Mace4 2005-2010.

[15] William McCune and Ranganathan Padmanabhan. Automated Deduction in Equational Logic and Cubic Curves. Springer-Verlag, Berlin, 1996.

[16] Ranganathan Padmanabhan and Sergiu Rudeanu. Axioms for Lattices and Boolean Algebras. World Scientific Publishers, 2008.

[17] Piotr Rudnicki and Josef Urban. Escape to ATP for Mizar. In First International Workshop on Proof eXchange for Theorem Proving-PxTP 2011, 2011.

[18] Stanisław Żukowski. Introduction to lattice theory. Formalized Mathematics, 1(1):215222, 1990.

Accepted June 30, 2021 